\title{
Effect of Energy Storages on Flexibility and Cyber Resilience of Electric Networks
}

\author{
Irina Kolosok, Elena Korkina*, Victor Kurbatsky \\ Melentiev Energy Systems Institute, Siberian Branch of Russian Academy of Sciences, Irkutsk, Russia
}

\begin{abstract}
When planning and managing the present-day and future transformed electric power systems (EPS), such comparatively new properties as flexibility and cyber resilience shall be taken into account along with EPS conventional properties, such as Reliability, Security, Survivability, and Vulnerability. Large-scale introduction of renewable energy sources notably lowers the EPS flexibility. Installation of Energy Storages allows compensation of power production imbalance occurred when using renewable energy sources, which makes the energy system more robust, but lowers its cyber security. The paper considers the main performances and models of Energy Storages, their impact on flexibility and cyber security of electric networks; it also presents the technique for quantifying the flexibility of a network with Energy Storages, and identifies most promising directions of studies in this area.
\end{abstract}

\section{Introduction}

In terms of generation, creation of SmartGrids implies development and integration of renewable energy sources (RES) [1], distributed generation and energy storage facilities under control of modern intelligent means. According to experts' estimates, use of RES in Russia by 2019 was to account for $15-18 \%$, but those predictions did not prove true. As of today, renewable energy sources in Russia make $2-3 \%$ of the total energy balance of the country.

One of the main causes of this lag is dependence of efficiency of the major RES types (solar and wind power plants) on the seasonal and weather conditions, i.e., power by these energy sources is generated irrespective of demand.

It raises uncertainty of planning the size of generating capacities, lowers stability and reliability of the energy systems, and worsens energy systems flexibility, one of their most important property. Flexibility is defined as ability of an energy system to keep and maintain normal operating conditions under the effect of internal (sudden changes in the loads, power flows in the links, and changes in the generation) and external (sudden disturbances) random (uncertain) factors [2-5 et al.].

Use of Energy Storages (ESs) is recognized to be one of the most efficient options of maintaining the power system flexibility as it allows leveling the imbalance of power generation when using RES, and make the system more sustainable.

In 2017 RF Ministry of Energy adopted the Concept of Developing the Energy Storage in Russian Federation [7]. Furthermore, milestones of implementing the pilot projects with the use of energy storages in Russia are indicated in the EnergyNet 'road map' within the longterm Program of National Technological Initiative.

Despite obvious advantages (higher EPS flexibility), large-scale use of Energy Storage raises a number of problems. One of them is reduction of cyber-resilience [8], which implies ability of a system to resist cyber attacks, to lower their initial negative impact, to adapt to them and to recover after them [9].

Planning and management of future transformed EPS requires account of these comparatively new properties (flexibility and cyber resilience). In this connection problems of flexibility and survivability analysis and maintenance require integrated and many-sided approach $[10,11]$.

The paper considers the main types of ESs used in Russia, their performances and models, impact of ESs on flexibility and cyber resilience of electric networks; consideration is also given to the technique for quantifying the network with ESs, to the most promising directions of studies on the issue.

\section{Main performances and types of ESs, areas of their application}

Electric energy storages are the most important components of developing SmartGrids, as they ensure [12]:

- Leveling the load curves in the network owing to electric power accumulation in the periods of excessive (cheap) power and its supply to the network in the periods of power shortage;

- Participation in frequency control (primary and secondary control);

- Integration of RES into the energy system;

\footnotetext{
*Corresponding author: korkina@ isem.irk.ru
} 
- Continuous power supply for major facilities, for auxiliary needs of power plants, substations, etc.

Main performances of Energy Storages [12]

Applicability of energy storage technologies in power industry is compared based on their performances that, in turn, depend on their physical properties. They include:

- Power is volume of power a storage facility can supply to the energy system;

- Energy capacity is energy a storage facility may accumulate and supply to the system;
- Time of response is the time required for an energy storage facility to transit from idle state (idle run, charging mode) to the mode of power supply of desired parameters;

- Time of discharge is time during which power is supplied to the energy system without additional charge.

Fig. 1 shows classification of energy storage facilities; Fig. 2 demonstrates fields of application of different types of energy storage facilities depending on their capacity, and period of accumulated energy discharge. Both figures have been borrowed from [13].

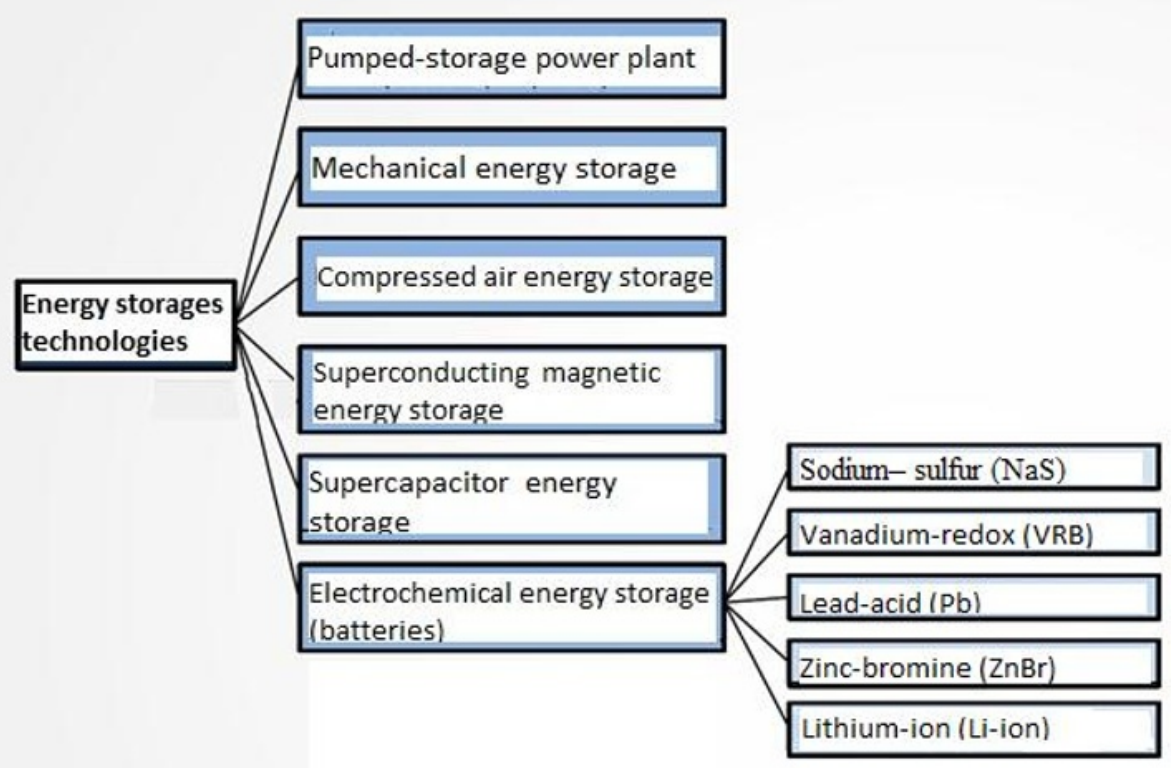

Fig. 1. Classification of energy storage facilities

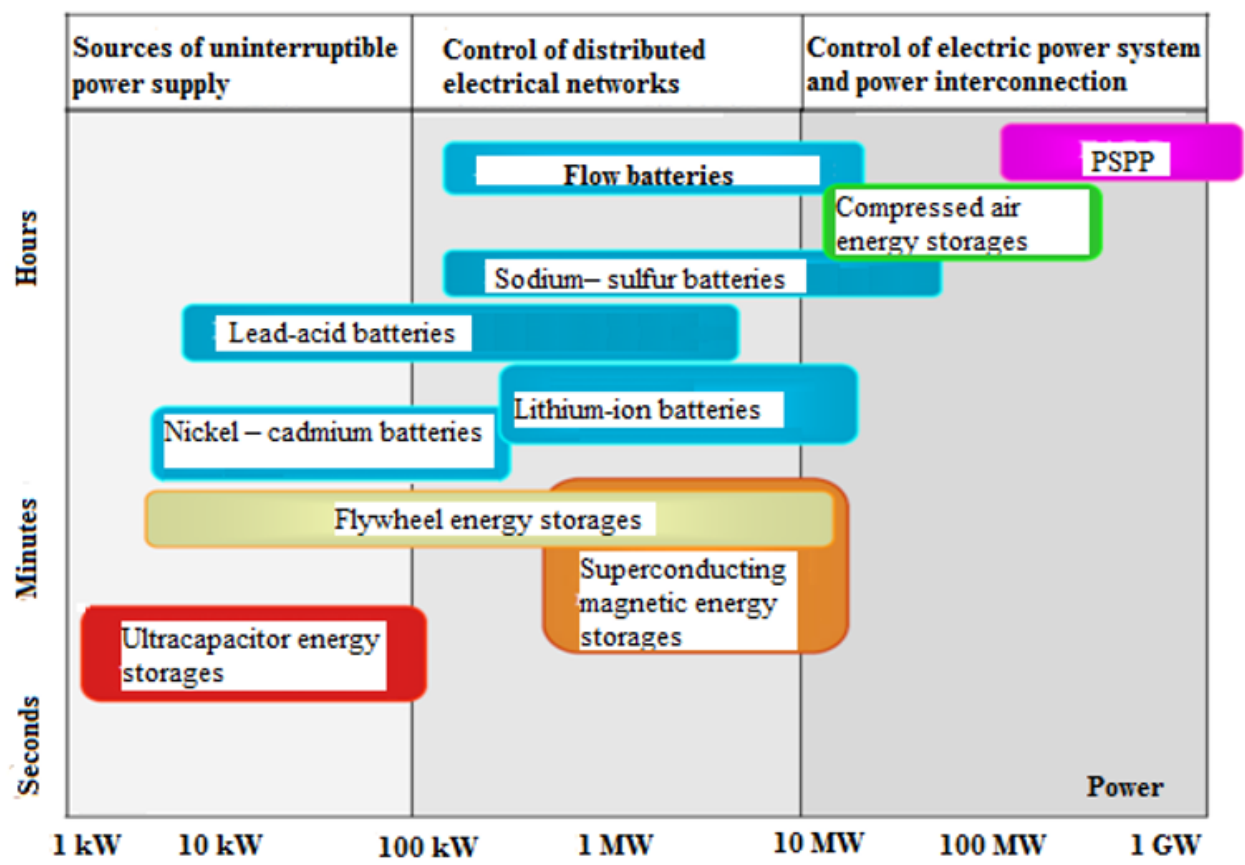

Fig. 2. Fields of application of different types of energy storage facilities 


\section{Model of a node with ESs for steady state calculation and estimating the EPS flexibility}

A model of a node with ESs that can be used for quantifying the EPS flexibility is proposed in Ref. [14, $15]$.

Dynamic behavior of some node $i$ with ESs can be approximately represented by a differential discrete in time first-order equation:

$$
C\left(x^{k, t+1}-x^{k, t}\right)=\eta_{l} P_{l o a d}^{k, t}-\frac{1}{\eta_{g}} P_{g e n}^{k, t}+\omega^{k, t}+v^{k, t}+\xi^{k, t}
$$

where $C$ - storage capacity, $x^{k, t}$ - state of charge of unit $k$ in period $t ; \eta_{l}, \eta_{g}$ - charge and discharge efficiencies; $P_{\text {gen }}^{k, t}$ - power production by unit $k$ in period $\mathrm{t} ; P_{\text {load }}^{k, t}-$ power consumption of unit $\mathrm{k}$ in period $t ; \omega, v, \xi$ - energy curtailment, storage losses, and consumption of primary energy carrier.

The left-hand part of equation (1) corresponds to the charge change, i.e., $0<x^{k, t} \leq 1$. The right-hand part of the equation represents exchange of real output between the facility and a network, the main fuel source $\xi$, and conditions for losses reduction.

The main processes described by equation (1) are demonstrated in Fig. 3 borrowed from [14].

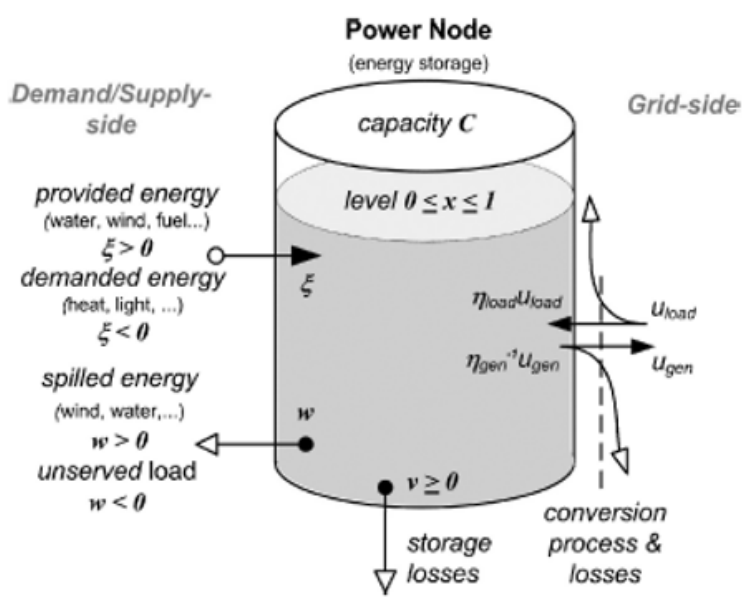

Fig. 3. Demonstration of exchange processes in the node with a storage facility.

\section{Quantification of flexibility of a network with ESs}

Large-scale integration of RES and variable schemes of transmission create high uncertainty in the energy system operation. Use of RES allows solution of a problem of power production and consumption, i.e., maintenance of their balance at every time moment, which considerably enhances flexibility of EPS with ESs. Still, operators of transmission systems need clear information on the available flexibility to be able to maintain the desired reliability level at reasonable costs.

Methodology of flexibility quantification for individual nodes of RES with ES and the network as a whole is proposed in [15]. For the purpose of quantification they introduce two notions: 1) Functional (operational) flexibility is ability of an energy system to absorb disturbances to be able to maintain steady-state operability; and 2) Territorial (localized) flexibility is functional flexibility available in a specific node of the network.

The methodology proposed allows expression of nodal flexibility in the terms $[R, P, E]$ :

$R$ - rate of some parameter change, e.g., it can be rate of accumulation (discharge) of generating equipment or ES;

$P$ - power;

$E$ - power constraints.

These three parameters are used to characterize nodal flexibility and identify a set of flexibility parameters (SET) that describe constraints of systems flexibility based on the contributions of each node with account of transmission limitations and power limitations that are of importance for nodes with Energy Storage. In other words, they describe the size of a disturbance in a certain node that can be compensated by appropriate and available recovery activities. These activities include redispatching measures, such as backup connection, participation of a consumer, changes in the network topology and in the specified power flow values.

The developed methodology includes the following steps:

Step 1: Universal modeling of flexibility of a single unit (component);

Step 2: Universal disturbance modeling;

Step 3: Network modeling;

Step 4: Identification of a set of parameters for flexibility quantifying;

Accomplishment of all the steps yields set $\mathrm{F}$ that expresses functional flexibility and contains all the possible values of $\delta$, such that there exists vector $\left(\delta, f_{s}\right)$ within the flexibility set

$$
F=\left\{\left(\delta, f_{s}\right) \in \mathfrak{R}^{n_{d}+n_{s}} \mid C_{s} f_{s}+C_{d} \delta \leq b\right\}
$$

where $C_{s}, C_{d}, b$ are packages (stacked versions) of systems constraints, accordingly; $\delta-$ a vector of variables related to disturbances; $f_{s}-$ a vector of variables characterizing flexibility. These vectors are stacked versions of the corresponding state variables of all units $k$ and all time steps $t$, expressed as

$$
f_{s}=\left[\begin{array}{l}
x \\
\Delta P \\
\Delta P_{\text {gen }} \\
\Delta P_{\text {load }}
\end{array}\right], \quad \delta=\left[\begin{array}{l}
\Delta R \\
\Delta P \\
\Delta E
\end{array}\right]
$$

Since a set of parameters characterizing flexibility $(\delta$, $f_{s}$ ) is known, it can be included into routine management by Systems Operator as an element of decision-making or as a means of monitoring/planning.

Using these data an operator of a transmission system can identify:

- The required flexibility to avoid foresighted disturbances; 
- A set of disturbances the system can overcome at specified available flexibility.

These problems have been stated in [1] et al. as problems of EPS Resilience Against Disturbances, including under cyber attacks.

\section{Cyber resilience of electrical networks with energy storages}

The cyber resilience of electrical networks with energy storages has much in common with such important properties as security and survivability that ensure the normal operation of electric power systems [16]. In the current context of the transition to Smart Grid, digitalization of energy, and increased cyber vulnerability of the entire power system and its individual infrastructures, in addition to these fundamental properties, there appears the property of cyber resilience, i.e. the ability of a complex technical (or information) system to exist and maintain its operability under cyberattack. Despite the seeming novelty of this feature, the measures required to provide it are in many respects similar to those developed and used to ensure the reliability and survivability of an electric power system $[17,18]$. These include the optimal placement of energy storage devices, redundancy, provision of power margins, etc., given the specific features of the tasks to be accomplished by using one or another energy storage system. A cyberattack on an energy storage device can lead to a partial or complete failure of the device itself, network control errors to the extent of serious accidents, and a decrease in the efficiency of the power system operation. Therefore, for example, the placement of the energy storage devices should meet the following main criteria [19]:

- minimization of the number and duration of customer disconnections in case of various accidents;

- minimization of the amount of electrical equipment (transformers, lines) to be replaced due to overcurrent in emergency conditions;

- minimization of losses in the electrical network by leveling the load curve and maintaining the voltage at the nodes;

- the most cost-effective demand-side response and power supply from energy storage devices, considering time-of-use rates

\section{Conclusions}

1. When planning and managing the present-day and future transformed EPS, such comparatively new properties as flexibility and cyber resilience shall be taken into account along with their traditional properties (Reliability, Security, Survivability, Vulnerability).

2. Challenge to develop the power generation methods that do not cause depletion of mineral fuel resources and to minimize environmental impact, originated a tendency towards larger use of RES, which became a vital necessity. Introduction of a larger number of RES and stepwise transition from centralized energy supply systems to distributed ones lower the energy system stability and reliability, and, which is more important, its flexibility, one of the most important features of energy systems.

3. Energy Storage is a key component in developing SmartGrids. Use of energy storage facilities ensures power production and consumption balance in the networks with RES thus providing the required flexibility level of energy system operation.

4. At the same time the use of ES reduces EPS cyber resilience, i.e., EPS ability to maintain workability under cyber attacks. Cyber resilience of networks with ES is a kind of guarantee of EPS robustness.

5. Perspective directions of studies in this field include: 1) implementation of the proposed technique for identifying the quantification metrics of flexibility; 2) development of methods for optimum ES allocation that would ensure required levels of flexibility and cyber resilience of present day and future Smart grid.

This study is supported by grant № 19-49-04108. "Development of Innovative Technologies and Tools for Flexibility Assessment and Enhancement of Future Power Systems".

\section{References}

1. https://ekoenergia.ru/oprobleme/vozobnovlyaemyie-istochniki-energii-vrossii.html

2. J. Cochran, M. Miller, O. Zinaman, M. Milligan, et al. Flexibility in 21st Century power systems // 21st Century Power Partnership, Denver,USA, Clean Energy Ministerial, (2014).

3. E. Lannoye, D. Flynn, M. O'Malley. Evaluation of power system flexibility // IEEE Trans. Power Syst., 27, 2, (2012).

4. U. Haeger, C. Rehtanz, N. Voropai. ICOEUR project results on improving observability and flexibility of large-scale transmission systems // IEEE PES General Meeting, San Diego, USA, (2012).

5. A. Ulbig, G. Andersson. Analyzing operational flexibility of electric power systems // 18th PSCC, Wroclaw, Poland, ( 2014).

6. Potapenko A.M., Melnikov V.D. Energy storage facilities in the electric power supply systems of industrial companies.

http://estorsys.ru/publications/sistemy-nakopleniyaenergii-v-sistemah

7. Concept of developing the market of energy storage facilities in RF, (2017).

https://minenergo.gov.ru/node/9013

8. Z. Wang, M.S. Nistor, S.W. Pickl. Analysis of the definitions of resilience // The 20th IFAC World Congress, Toulouse, France, (2017).

9. C. Nan, G. Sansavini, W. Kroeger. Building an integrated metric for quantifying the resilience of interdependent infrastructure systems // 9th Intern. 
Conf. on Critical Information Infrastructure Security, Limassol, Cyprus, (2014).

10. Massoud A. Challenges in reliability, security, efficiency, and resilience of energy infrastructure: Toward smart self-healing electric power grid // IEEE PES General Meeting, Pittsburg, USA, (2008).

11. N. Voropai, I. Kolosok, E. Korkina, A. Osak. Transformation of properties of future energy systems and problems of ensuring their steady-state operation. Baku, (2018).

12. Yu. Kulikov. Electric energy storages as an efficient tool for managing the operating conditions of electric power systems.

13. N. Novikov, A. Novikov. Prospects of using the energy storage and intelligent management systems for distributed generation with involvement of renewable energy sources. OJSC NTC FSK EES, (2012).

14. K. Heussen, S. Koch, A. Ulbig, G. Andersson Unified System-Level Modeling of Intermittent Renewable Energy Sources and Energy Storage for
Power System Operation // 140 IEEE Systems Journal, 6, 1, (2012).

15. M.A. Bucher, S. Delikaraoglou, K. Heussen, P. Pinson, G. Andersson. On quantification of flexibility in power systems, IEEE PowerTech, Eindhoven, Netherlands, (2015).

16. N. Voropai, I. Kolosok, E. Korkina, A. Osak. Problems of vulnerability and survivability of cyberphysical and electric power systems // Energy Policy, 5, (2018).

17. N. Voropai. EPS Survivability: Methodological grounds and methods of studies // Izv. AN SSSR. Energy and transport, 6, (1991).

18. A. Diakov, V. Stennikov, S. Senderov, M.Sukharev et al. Reliability of energy systems: Problems, models and methods of their solution (Editor N. I. Voropai. - Novosibirsk: Nauka, 2014).

19. V. Stroev, A. Gremiakov, C. Arachchige, Z.Styczynski. Optimal Allocation of Energy Storage devices in electrical power systems. 13-th PSCC in Trondheim, (1999) 